\title{
Fatores de risco da prematuridade: uma revisão narrativa
}

\author{
Risk factors for prematurity: a narrative review
}

Factores de riesgo de prematuridad: una revisión narrativa

Alef Alioscha Andrade Maia ${ }^{1 *}$, Ana Paula Oliveira Pinto ${ }^{1}$, Juliana Nascimento Viana ${ }^{1}$, Gabriela Amaral de Sousa1', Giovanna Guimarães Mourão¹.

\section{RESUMO}

Objetivo: Discorrer sobre os fatores de risco de prematuridade. Revisão bibliográfica: A gravidez é um período em que a mulher é acometida a alterações no seu corpo sendo mudanças endócrinas metabólicas. Em gestações normais esse período por chegar à40 semanas, já em período entre 37 e 38 semanas e seis dias é conceituada com o termo precoce e pode apresentar maiores complicações, desencadeando um parto prematuro. A prematuridade é ponderada com a maior causa de morte em crianças nos primeiros cinco anos de vida no Brasil e como ausência de pré-natal, o uso de drogas licitas e ilícitas, abortos espontâneos após o primeiro trimestre gestacional; idade materna inferior a 15 anos ou superior a 40 anos; gestação múltipla e ausência de controle pré-natal, e problemas crônicos de saúde da mãe. Considerações finais: A prematuridade é uma dificuldade na saúde pública, por isso é importante que haja o preparo do complexo do sistema de saúde, e que consiga prestar todo o atendimento de acordo com as necessidades das gestantes. O pré-natal é uma ferramenta para minimizar os índices, pois é através do acompanhamento da gestação é que pode identificar as características que representam risco e assim instituir medidas para a sua prevenção.

Palavras-chave: Fatores de risco, Parto prematuro, Sinais e sintomas.

\begin{abstract}
Objective: To discuss the risk factors for prematurity. Bibliographic review: Pregnancy is a period in which the woman is affected by changes in her body, being metabolic endocrine changes. In normal pregnancies, this period can reach 40 weeks, in the period between 37 and 38 weeks and six days it is conceptualized with the early term and can present greater complications, triggering a premature birth. in the first five years of life in Brazil and as a lack of prenatal care, the use of licit and illicit drugs, spontaneous abortions after the first gestational trimester; maternal age below 15 years or above 40 years; multiple pregnancy and lack of prenatal control, and chronic health problems for the mother. Final considerations: Prematurity is a difficulty in public health, so it is important to prepare the health system complex, and to be able to provide all the care according to the needs of pregnant women. Prenatal care is a tool to minimize the rates, because it is through the monitoring of pregnancy that you can identify the characteristics that represent risk and thus institute measures for their prevention.
\end{abstract}

Keywords: Risk factors, Obstetric labor premature, Signs and symptoms.

\section{RESUMEN}

Objetivo: Discutir los factores de riesgo para la prematuridad. Revisión bibliográfica: El embarazo es un período en el que la mujer se ve afectada por cambios en su organismo, siendo cambios metabólicos endocrinos. En embarazos normales, este período puede alcanzar las 40 semanas, en el período entre 37 y

${ }^{1}$ Universidade Federal do Amazonas (UFAM), Manaus - AM. *E-mail: alef-maia@hotmail.com 
38 semanas y seis días se conceptualiza con el término precoz y puede presentar mayores complicaciones, desencadenando un parto prematuro en los primeros cinco años de vida en Brasil y como la falta de atención prenatal, el uso de drogas lícitas e ilícitas, los abortos espontáneos después del primer trimestre de gestación; edad materna menor de 15 años o mayor de 40 años; embarazo múltiple y falta de control prenatal, y problemas crónicos de salud de la madre. Consideraciones finales: La prematuridad es una dificultad en salud pública, por lo que es importante preparar el conjunto del sistema de salud, y poder brindar todos los cuidados acordes a las necesidades de las gestantes. El control prenatal es una herramienta para minimizar los índices, ya que es a través del seguimiento del embarazo que se pueden identificar las características que representan riesgo y así instituir medidas para su prevención.

Palabras clave: Factores de riesgo, Trabajo de parto prematuro, Signos y síntomas.

\section{INTRODUÇÃO}

A gravidez pode ser descrita como um momento que é vivenciado na vida da mulher que requer a transição em um período de tempo e que é considerada como o processo normal de desenvolvimento, envolvendo transformação de identidade e nova definição de papéis. E são nessas modificações do organismo que podem ser estabelecidas mudanças que tem como papel primordial promover e beneficiar a saúde de mãe e filho e quando exigências não são atendidas podendo suceder prejuízos relacionados ao prognóstico materno e perinatal. No caso das gestações de mulheres primípara, é que além de ser o primeiro parto é também o momento em que vai ocorrer a mudança do processo de passar de filha e de mulher, é assim iniciar o processo de ocupar o lugar de mãe (DE FELICE EM, 2000).

Durante todo o período gestacional é possível evidenciar que irá ocorrer alterações endócrinas metabólicas, visando assim promover e atender as necessidades materno-fetais, tendo o colo uterino papel central na manutenção da gestação (LARMA JD e IAMS JD, 2012). São modificações que ocorrem no organismo da mulher e que podem também desencadear diversos sentimentos não positivos, e em contrapartida é uma parte da vida e que para algumas gestantes é considerado especial todas as etapas, mas em alguns eventos a gestação pode ter algumas ocorrências indesejadas e neste caso épreciso considerar as alterações gestacionais (CAMACHO KG, et al., 2010).

É de extrema importância ressaltar, que a gravidez entre trinta e sete e trinta e oito semanas e seis dias conceituada como termo precoce, apresenta maiores complicações do que as que atingem entre trinta e nove semanas e quarenta semanas e seis dias (termo completo). A prematuridade é qualificada conforme a Idade Gestacional (IG) do Recém-Nascido (RN). Segundo Freitas F, et al. (2011), em sua pesquisa discorreu que a prematuridade moderada pode ser referenciada àqueles $R N s$ que tenham nascidos entre trinta e duas e trinta e seis semanas de IG, prematuridade acentuada para os nascidos entre vinte e oito e trinta e uma semanas de IG e prematuridade extrema para aqueles que nasceram com IG inferior a vinte e oito semanas (ALMEIDA TSO, et al., 2013).

O parto prematuro é determinado e expresso como o nascimento antes do termo, sendo assim, fetos que nascem anteriormente a maturidade fetal. Sua classificação se dá por ordem de acontecimentos: nascimento que antecede trinta e sete semanas de gestação, que são calculadas a partir do primeiro dia da última menstruação, desta forma é possível discorrer que o parto prematuro pode ter consequências obstétricas para o parto, prevendo riscos maternos, incluindo distúrbios hipertensivos, deslocamento prematuro da placenta ou complicações fetais que impedem o desenvolvimento intrauterino e causam sofrimento fetal, com média de $25 \%$ dos casos (MONTENEGRO RF, 2008).

Nesta vertente o parto prematuro é distinguido como parto do $\mathrm{RN}$ com menos de trinta e sete semanas ou o equivalente menos que trezentos e cinquenta e nove dias, e de acordo com o relato de França EB, et al. (2017) a prematuridade é contemplada como a alicerce da causa de morte em crianças nos cinco anos iniciais de vida no Brasil, e dessa forma é imprescindível que existam diálogos para estabelecerem abordagens para sua prevenção. 
Não obstante aos avanços que já foram evidenciados na obstetrícia, a quantidade de nascimentos de prematuros é expressivamente considerada elevado, especialmente em países subdesenvolvidos e em progresso de evolução, como o Brasil, é possível assinalar uma tendência de aumento dos casos (SILVEIRA MF, et al., 2010).

A sua prevalência pode apresentar variações que oscilam de acordo com as características da população, mas em âmbito geral, mostra um aumento no mundo nos últimos anos. No registro de Chawanpaiboon S (2019), ele discorre que no Brasil, a prevalência chega a 12\% (entre 10\% e 14\%) registrando 3.000 .000 de nascidos vivos tendo seu nascimento antes das 37 semanas. Esse registro comprova que cerca de 360 mil crianças nascem prematuras no ano, chegando a quase 1000 crianças ao dia, sendo que $70 \%$ ocorrem nos primeiros vinte e oito dias de vida neonatal, quando analisado a quantidade nascimentos prematuros é possível evidenciar que na América Latina, cerca de 12 milhões de crianças nascem a cada ano, e 135.000 delas morrem ou 11,5 por 1.000 nascidos vivos por ser prematuro (UNITED NATIONS INTERNATIONAL CHILDREN'S EMERGENCY FUND, 2013).

$\mathrm{Na}$ visão de Blencowe $\mathrm{H}$, et al. (2013), quando avaliado sobre as questões da incidência o Brasil encontra-se no ranking em nono lugar com maior índice de nascimentos prematuros o que pode ser classificado como um problema de saúde pública, e que algumas condições que são dispostas pela mãe podem favorecer o risco durante o período gestacional.

O uso de drogas ilícitas durante a gravidez pode acarretar diversos danos ao feto, o mesmo é descrito quando a mãe faz o uso do consumo do álcool durante o período da gestação, pois está associado a diversos efeitos teratogênicos. Durante esse tempo de gravidez fazer ter o hábito de consumir substâncias alcoólicas pode impedir e dificultar o crescimento intra-uterino. Já quando relatado sobre o tabaco é possível identificar a presença de substâncias que podem atravessar a barreira placentária, como monóxido de carbono e nicotina. O monóxido de carbono danificar a hemoglobina fetal, desencadeando o impedimento a ligação do oxigênio e causa hipoxemia fetal, o que pode causar o retardo do crescimento intrauterino e o descolamento prematuro da placenta. É válido ponderar que as substâncias que são presentes no tabaco podem ser excretadas do leite materno e reduzir a secreção de leite, uma vez que as substâncias da cannabis podem atravessar a barreira placentária, prejudicar o crescimento do feto e também atingir o sistema nervoso fetal (BORSON LAMG, et al., 2019).

Já Yamaguchi ET, et al. (2008) no seu estudo enfatizaram que além de cruzar a barreira placentária para atingir a vascularização fetal, o uso de cocaína também pode causar o descolamento prematuro da placenta.

O nascimento de um prematuro representa um fator de extrema preocupação para a saúde do recémnascido, pois leva à sua mortalidade e morbidade. No longo prazo, os bebês prematuros têm maior probabilidade de ter consequências graves para a saúde, como cegueira, paralisia cerebral, problemas de aprendizagem e desenvolvimento e que a mortalidade neonatal responde por aproximadamente $70 \%$ da mortalidade infantil no país, sendo a prematuridade a principal causa de morte neonatal em todas as regiões do Brasil; aponta questões assistenciais relacionadas ao pré-natal, ao parto e ao recém-nascido (GWEN LATENDRESSE CNM, 2009).

De acordo com Rattasumpun P e Raines DA (2008), ainda que a prematuridade promova um grande e vasto impacto nas mulheres que já vivenciaram tal situação com seus filhos, este fato pode ocorrer em um momento avaliado como parte do ciclo de vida saudável, a assumir o papel de doentes que necessitam de cuidados especiais, e além de esse sofrimento se estender até o período puerperal (BRANDON DH, et al., 2011).

Diante do exposto e considerando a importância do assunto relacionados aos fatores de risco de prematuridade, buscou-se como objetivo realizar por meio de uma revisão narrativa discorrer sobre as condições que podem ocasionar intercorrências no período gestacional, e desta forma elucidar os possíveis questionamentos relacionados a este tema. 


\section{REVISÃO BIBLIOGRÁFICA}

No Brasil, conforme dados do DataSus, em 2019, já foram anotados mais de 315 mil casos de nascimentos de prematuros (BRASIL, 2019). Os fatores de risco já relacionados e descritos pelo Ministério da Saúde, discorrem sobre a evidência à história obstétrica, consistindo em: parto prematuro prévio; história de perdas de um ou mais abortos espontâneos após os três meses, idade materna no mínimo quinze anos ou maior que quarenta anos; gestação múltipla, ou falta de controle pré-natal (BRASIL, 2012).

Em proporções mundiais é possível ressaltar que um a cada dez nascimentos são considerados prematuro no ano de 2010, e nesse contexto são estimados o nascimento de 15 milhões recém-nascido. Fato este que representa uma taxa de $11,1 \%$ dos nascimentos, com um milhão de óbitos em decorrência direta do nascimento prematuro (WORLD HEALTH ORGANIZATION, 2015).

Quanto à prematuridade, muitos autores em diversos estudos entram em consenso que as mulheres que apresentam idade mais extremas retratam maior índice de ser acometidas as complicações gestacionais e implicações da evolução gestacional e resultado neonatal (SHRIM A, et al., 2011).

Partindo desta colocação quanto aos fatores de risco os mesmos são relatados por Potter PA e Perry AG, (2013), onde estes podem ser classificados em categorias que podem ser inter-relacionadas a: fatores genéticos e fisiológicos que abrangem o funcionamento físico, as questões de cargas ou predisposição genética a uma patologia; a faixa etária que ocorre com mais suscetibilidade da pessoa, e as questões relacionada ao ambiente e quais as condições desta área (ar, água e solo) e que se vive.

As circunstâncias que estimulam o Trabalho de Parto Prematuro (TPP) podem estar associadas a multifatores dentre eles: epidemiológicos, obstétricos e ginecológicos, não deixando de evidenciar os fatores clínico-cirúrgicos, como as doenças maternas, infecções geniturinárias e processos cirúrgicos que sã realizados durante a gravidez (ROOS C,et al.,2015).O parto pode ocorrer prematuramente de maneira espontânea ou eletiva, quando existe a indicação médica por intercorrências maternas e/ou fetais (MARRA NBF, et al., 2016).

O nascimento prematuro é uma das condições central de morbidade e mortalidade infantil. Em 2013, $36 \%$ das 8.470 mortes de bebês nos Estados Unidos foram atribuídas ao nascimento prematuro. (SHAPIRO-MENDOZA CK,et al., 2016).

As questões quando assinaladas ao baixo nível socioeconômico materno é, ainda hoje, um dos principais fatores de risco para a antecipação do parto, muito devido à subnutrição, às infecções e à falta de assistência pré-natal adequada (ALMEIDA AC, et al., 2012).

As consequências da prematuridade representam grave problema de saúde pública. No ano de 2004 já começavam a destacar o quanto a importância a atenção às grávidas com visão política governamental se fazia fundamental e que já se encontrava promulgada no conjunto de normas que conduzem a atuação do Sistema Único de Saúde (SUS) (LEAL M, et al., 2004).

A etiologia do parto prematuro pode ser apreciada como multifatorial, sendo comprometida por condições ambientais e genéticas agindo como contribuintes de riscos, comumente classificados como primários quando há presença antes da gravidez, ou secundários, quando começam seu desenvolvimento no curso da gestação (LIMA JDA, et al., 2012).

Segundo relatado por Melo E, et al., (2015) quanto ao entendimento relacionados a consultas no prénatal, seria possível ter um controle maior no número de partos prematuros, por meio de uma assistência de qualidade e eficiências, buscando fazer todo o monitoramento especifico de cada gestante, e por meio desta anamnese conseguiria identificar os possíveis risco de haver um parto antes do tempo estabelecido.

No ano de 2011, já era possível afirmar que quase $60 \%$ das mães de prematuros durante todo o período gestacional compareciam em menos de cinco consultas do pré-natal, e essas mães depararam com chances cinco vezes a mais de desencadear um processo de prematuridade quando realizada a comparação com mães de crianças a termo (FERRAZ RT e NEVES ET, 2011). 
Apesar de ter sido possível visualizar a evolução tecnológica e científica que ocorreu nas últimas décadas em relação à parte obstétrica, a prematuridade ainda é considerada um fator que constitui um grande desafio quando observado em âmbito geral e nesse sentido é a principal causa de morbidade e mortalidade neonatal, além de ser considerada uma das principais causas de morte em crianças com menos de um ano. Quando é relatado a relação entre a morbilidade e a mortalidade neonatal e as possíveis e diversas sequelas que o RN prematuro possa vir a apresentar durante sua infância, adolescência e vida adulta, as mesmas estão diretamente correlacionadas e associadas com a IG de nascimento. É importante dizer que a prematuridade extrema pode ser considerada quando é descrito recém-nascido com menos de vintee oito semanas de IG é a que está mais associada a injúrias, tanto neonatais quanto tardias (BLENCOWE H, et al., 2012).

A base decisiva do risco de óbito para o neonato pode ser evidencias no nascimento com baixo peso e a prematuridade, e partindo desse princípio a maneira mais adequada de se reduzir ou minimizar a taxa de mortalidade e as dificuldades em relação às sequelas que se desencadeiam nesses é buscar na grande maioria dos partos deixar que ocorra nascimento de prematuros, sendo a realização de anamnese de forma bem completa em todas as consultas e até mesmo no contexto histórico de vida antes da gestação a primeira etapa para evidenciar a mulher com risco para o parto prematuro (GONZAGA ICA, 2016).

Todo o processo de investigação da saúde da gestante é realizado por meio da anamnese, e desta forma é possível evidenciar os inúmeros fatores que podem desencadear uma gravidez ao risco, e entre elas podem estar às classificações por risco estatístico, social, pessoal ou de identificação inicial. A faixa etária materna tem sido ponderada como condição de risco quando a gravidez não ocorre na idade ideal, sendo assim, nos extremos da vida reprodutiva, neste casos pode ser tanto para gestações muito imaturas ou em muitos casos tardias, mas na grande maioria dos relatos e que os riscos da gravidez precoce tem sido comprovada como grave problema de saúde pública (CABRAL RA, et al., 2017).

Gravidez na adolescência ou até mesmo quando são evidenciadas antes dos dezenove anos inspira e requer um maior cuidado e atenção, pois podem promover e desenvolverem com possíveis implicações que são prejudiciais à saúde da mãe e da criança. Buscando estabelecer um melhor entendimento podem ser assinalados os riscos para o concepto devido ao: baixo peso ao nascer, carências de micronutrientes e reserva do aumento intrauterino, e que pode ocasionar modificações no progresso da gestação e assim promover implicações que desencadeie em um possível parto prematuro. No âmbito geral, é importante estabelecer um cuidado distinguido de acordo com as características anátomo-fisiológicas dos RNs prematuros quando confrontada sãos RNs a termo. Pois, os nascidos pré-termo apresentam maior susceptibilidade agravos de saúde, haja vista o seu incompleto desenvolvimento fetal e de sua maior sensibilidade às infecções (GUIMARÃES E, et al., 2017).

Quando foi avaliado quais seriam os possíveis agravantes de complicações neonatais as que mais são descritas são Síndrome da Angústia Respiratória (SAR), hemorragia intracraniana, enterocolite necrosante e morte neonatal. Esses achados de intercorrências são mais constantes em idades gestacionais inferiores há trinte e duas semanas, tornando-se críticas abaixo de vinte e oito semanas (BITTAR RE, 2018).

Outro fator ligado aos bebês prematuros é o peso ao nascer, que pode ser caracterizado como baixo, em decorrência de infecções do trato geniturinário, baixa estatura materna, falta de acompanhamento de prénatal, déficits nutricionais ou doença periodontal. Os fatores de risco de parto pré-termo parecem ser semelhantes aos fatores de risco para as doenças periodontais (tabaco, etnia, níveis socioeconômicos e educacionais) e podem confundir a associação entre periodontite e parto prematuro (HUCK O, et al., 2011).

Segundo Doria MT e Spautz CC (2011), os RNs nascidos prematuros podem necessitar de cuidados especializados tanto no período neonatal como também mais tardiamente na sua vida escolar, principalmente devido ao risco de apresentarem complicações precoces e sequelas no futuro.

As manifestações clínicas que ajudam a identificar o parto prematuro são sinais ou sintomas como contrações a cada dez minutos ou mais, cólicas no baixo ventre ou semelhantes a cólicas menstruais, presença ou alterações no corrimento vaginal, pressão pélvica, lombalgia, dor abdominal cólicas com ou 
sem diarréia, aumento da pressão pélvica ou vaginal e até sangramento vaginal leve podem identificar o início do trabalho de parto, e um histórico médico detalhado é o primeiro passo para identificar mulheres em risco de trabalho de parto prematuro. O tabagismo no período gestacional é considerado o principal fator de risco apresentando desfechos desfavoráveis, tanto para o bebê quanto para a mãe. Fato este pode ser representado por cerca de $5 \%$ a $8 \%$ dos partos prematuros, quanto ao nascimento de baixo peso é possível ter uma média de $13 \%$ a $19 \%$ e por fim $5 \%$ a $7 \%$ da síndrome da morte súbita do lactente (DIETZ PM, et al., 2009).

Quando discorrido e abordado sobre as possíveis manifestações clínicas estas podem ser evidenciadas e são de grande valia para que se através destas se consiga realizar toda a investigação durante o pré-natal e assim ajudar a identificar o parto prematuro, e nessa vertente e diante de todos os relatos acima descrito é imprescindível que as gestantes passem pela consultas mensais, e todo o acompanhamento necessário estabelecido pelo médico pois são nesse encontro junto ao médico que é possível identificar quais são as possibilidades ou até mesmo verificar quais são os fatores de riscos eminente que a paciente e o bebê podem desencadear durante os mesmo, e já conseguir precisar quais as possíveis intervenções que podem ser realizadas por parte dos profissionais de saúde (DAMÉ JLD, et al., 2019).

Está bem fundamentado pela literatura científica que o tabagismo durante a gestação se associa a restrição do crescimento intrauterino e consequentemente menor peso e comprimento ao nascer, por outro lado as repercussões em longo prazo da exposição ao tabagismo durante o crescimento infantil são menos definidas (MURARO AP, et al., 2015)

Possato $\mathrm{M}$, et al. (2007) realizaram estudo onde investigaram 27 gestantes fumantes que foram atendidos em um hospital que atende gestantes de alto risco no interior de São Paulo e após todo o período de investigação concluíram que predomina a baixa escolaridade; têm entre vinte e trinta e seis anos (70,4\%); $70,3 \%$ são donas de casa, 3,7\% cozinheiras, $14,8 \%$ faxineiras, $3,7 \%$ secretárias e $7,4 \%$ escriturárias e $33,3 \%$ são de primeiro parto. Portanto, observa-se que os principais fatores relacionados ao tabagismo materno são: baixo nível socioeconômico e baixa escolaridade, pois outros estudos com gestantes fumantes também comprovaram esses mesmos resultados.

\section{CONSIDERAÇÕES FINAIS}

Devido às diversas correlações que existem entre os muitos fatores de risco que podem ser assinalados e descritos durante o período gestacional, o nascimento prematuro é considerado como um problema complexo de saúde pública, pois mesmo com a evolução da área obstétrica, ainda a lacunas para serem descobertas, em virtude da morbimortalidade neonatal que causa. É imprescindível que seja realizado novos estudos afim de buscar maior conhecimento correlacionados aos fatores de prematuridade objetivando um diagnóstico em muitos casos precoce, ou quando houver necessidade realizar um acompanhamento contínuo, buscando prevenir as possíveis complicações durante o período gestacional.

\section{REFERÊNCIAS}

1. ALMEIDA AC, et al. Fatores de risco maternos para prematuridade em uma maternidade pública de Imperatriz -MA. Rev Gaucha de enfermag, 2012; 33(2): 86-94.

2. ALMEIDA TSO, et al Investigação sobre os Fatores de Risco da Prematuridade: uma Revisão Sistemática. R brasci Saúde, 2013; 17(3): 301-308

3. BITTAR RE. Parto pré-termo. Rev Med, 2018; 97(2): 195-207.

4. BLENCOWE H, et al. Born too soon: the global epidemiology of 15 million preterm births. Reprod Health, 2013 ; 10 Suppl 1: S2.

5. BLENCOWE H, et al. National, regional, and worldwide estimates of preterm birth rates in the year 2010 with time trends since 1990 for selected countries: a systematic analysis and implications. Lancet, 2012; 379(9832): $2162-72$.

6. BORSON LAMG, et al. A exposição de substâncias na gestação e lactação. Revista Saúde em Foco, $2019 ; 11$.

7. BRANDON DH, et al. Emotional responses of mothers of late-preterm and term infants. J Obstet Gynecol Neonatal Nurs. 2011; 40(6): 719-31.

8. BRASIL. DataSus. Relação de nascimentos de recém-nascidos pré-termo e baixo peso no ano de 2019. Disponível em: http://tabnet.datasus.gov.br/cgi/deftohtm.exe?sinasc/cnv/nvuf.def. Acesso em: 19 de janeiro de 2022. 
9. BRASIL. Gestação de alto risco: manual técnico. Ministério da Saúde, Secretaria de Atenção à Saúde, Departamento de Ações Programáticas Estratégicas. 2012. Disponível em: https://bvsms.saude.gov.br/bvs/publicacoes/gestacao_alto_risco_maual_tecnico_4ed.pdf. Acessado 19 de janeiro de 2022.

10. CABRAL RA, et al. A experiência de ser mãe pela primeira vez após os 35 anos. Revista Cinergis, 2017; 18(4): 279284.

11. CAMACHO KG, et al. Vivenciando repercussões e transformações de uma gestação: perspectivas de gestantes. Ciencia y Enfermeria.Concepcion, 2010; 16(2): 115-125.

12. CHAWANPAIBOON S, et al. Global, regional, and national estimates of levels of preterm birth in 2014: a systematic review and modelling analysis. Lancet Glob Health, 2019;7: 37-46

13. DAMÉ JLD, et al. Cessação do tabagismo na gestação: estudo de base populacional. Rev Saude Publica, 2019;53:3

14. DE FELICE EM. A Psicodinâmica do Puerpério.São Paulo: Editora Vetor, 2000.

15. DIETZ PM, et al. Infant morbidity and mortality attributable to prenatal smoking in the U.S. Am J Prev Med, 2009; 114(2 Pt 1): 318-25.

16. DORIA MT, SPAUTZ CC. Trabalho de parto prematuro: predição e prevenção. Rev. Femina, 2011; 39(9): 443-449

17. FERRAZ RT, NEVES ET. Fatores de risco para baixo peso ao nascer em maternidades públicas. Rev Gaúcha Enferm, 2011; 32(1): 86-92.

18. FRANÇA EB, et al. Principais causas da mortalidade na infância no Brasil, em 1990 e 2015: Estimativas do Estudo de Carga Global de Doença. Ver Bras Epidemiol, 2017; 20(Suppl 01): 46-60.

19. FREITAS F, et al. Rotinas em obstetrícia. 6ª ed. Porto Alegre: Artmed, 2011; 902p.

20. GONZAGA ICA, et al. Atenção pré-natal e fatores de risco associados à prematuridade e baixo peso ao nascer em capital do nordeste brasileiro. Ciência \& Saúde Coletiva, 2016; 21(6): 1965-1974.

21. GUIMARÃES E, et al. Prevalência e fatores associados à prematuridade em Divinópolis, Minas Gerais, 2008-2011: análise do Sistema de Informações sobre Nascidos Vivos.Revista Epidemiológica Serv Saúde, 2017; 26(1):91-98.

22. GWEN LATENDRESSE CNM. The interaction between chronic stress and pregnancy: preterm birth from a biobehavioral perspective. J Midwifery Womens Health, 2009; 54(1):8-17.

23. HUCK O, et al. Relation ship between Periodontal Diseases and Preterm Birth: Recent Epidemiological and Biological Data. Journal of Pregnancy, 2011.

24. LARMA JD, IAMS JD. Is sonographic assessment of the cervix necessary and helpful? Clin Obstet Gynecol, 2012; $55(1): 324-35$.

25. LEAL M, et al. Uso do índice de Kotelchuck modificado na avaliação da assistência pré-natal e sua relação com as características maternas e o peso do recém-nascido no Município do Rio de Janeiro. Cad Saúde Pública, 2004; 20(Supl. 1): S63-S72.

26. LIMA JDA, et al. Fatores de risco relacionados à prematuridade ao nascer: um estudo caso-controle. Odonto, 2012; 20(40): 119-127.

27. MARRA NBF, et al. Prematuridade eletiva e as suas repercussões perinatais nas síndromes hipertensivas da gestação. Rev UNILUS Ens e Pesq, 2016; 13(32): 26-32.

28. MELO E, et al. Fatores associados à qualidade do pré-natal: uma abordagem ao nascimento prematuro. Revista Escola de Enfermagem, 2015; 49(4): 540-549.

29. MONTENEGRO RF. Obstetrícia fundamental. 11aㅡ ed. Rio de Janeiro: Guanabara Koogan,2008.

30. MURARO AP, et al. Effect of the exposure to maternal smoking during pregnancy and childhood on the body mass index until adolescence. Rev Saúde Pública, 2015; 49.

31. POSSATO M, et al. Representação de gestantes tabagistas sobre o uso do cigarro: estudo realizado em hospital do interior paulista. Rev. Escola de Enfermagem USP, 2007; 41: 3.

32. POTTER PA, PERRY AG. Fundamentos de Enfermagem. Elsevier, 2013.

33. RATTASUMPUN P, AINES DA. The experience of Thai women facing the onset of preterm labor. MCN Am J Matern Child Nurs, 2008; 33(5): 302-6.

34. ROOS C, et al. Predictive Factors for Delivery within 7 Days after Successful 48-Hour Treatment of Threatened Preterm Labor. AJP, 2015.

35. SHAPIRO-MENDOZA CK, et al. CDC grand rounds: public health strategies to prevent preterm birth. Morbidity and Mortality Weekly Report (MMWR),2016; 65(32): 826-830.

36. SHRIM A, et al. Is young maternal age really a risk factor for adverse pregnancy outcome in a Canadian tertiary referral hospital? J Pediatr Adolesc Gyneco, 2011; 24(4): 218-222.

37. SILVEIRA MF, et al. Determinantes de nascimento pré-termo na coorte de nascimentos de 2004, Pelotas, Rio Grande do Sul, Brasil. Cad Saúde Pública, 2010; 26(1): 185-94.

38. UNITED NATIONS INTERNATIONAL CHILDREN'S EMERGENCY FUND. Estudo faz alerta sobre a situação da prematuridade no Brasil. 2013. Disponível em: https://memoria.ebc.com.br/infantil/para-pais/2013/09/estudo-fazalerta-sobre-a-situacao-da-prematuridade-no-brasil. Acesso em: 25 de janeiro de 2022.

39. WORLD HEALTH ORGANIZATION. Preterm birth, 2015. Disponível em: http://www. who.int/mediacentre/factsheets/fs363/en/. Acesso em: 25 de janeiro de 2022.

40. YAMAGUCHI ET, et al. Drogas de abuso e gravidez. Drogas de abuso e gravidez. Revisão da Literatura. Arch. Clin. Psychiatry, 2008; 35(suppl 1). 